PROCEEDINGS OF THE AMERICAN MATHEMATICAL SOCIETY

Volume 124, Number 5, May 1996

\title{
GEOMETRY OF KODAIRA MODULI SPACES
}

\author{
SERGEY A. MERKULOV \\ (Communicated by Christopher B. Croke)
}

\begin{abstract}
A general theorem on the existence of natural torsion-free affine connections on a complete family of compact complex submanifolds in a complex manifold is proved.
\end{abstract}

\section{INTRODUCTION}

Let $X$ be a compact complex submanifold of a complex manifold $Y$. The chief object of interest in this paper is the set $M$ of all holomorphic deformations of $X$ inside $Y$, i.e. a point $t$ in $M$ can be thought of as a "nearby" compact complex submanifold $X_{t}$ in $Y$. The first natural question about this set - when is it a manifold? — was answered by Kodaira [9] in 1962. He found a surprisingly simple sufficient condition, the vanishing of the first cohomology group $H^{1}(X, N)$ with coefficients in the normal sheaf $\left.N \equiv T Y\right|_{X} / T X$, for $M$ to have an induced complex manifold structure. Moreover, he established a clear correspondence between the first floors of two towers of infinitesimal neighbourhoods generated by embeddings, $t \hookrightarrow M$ and $X_{t} \hookrightarrow Y$, by proving that there is a canonical isomorphism $\mathrm{k}_{t}$ : $T_{t} M \longrightarrow H^{0}\left(X_{t}, N_{t}\right)$ which associates a global section of the normal bundle $N_{t}$ of $X_{t} \hookrightarrow Y$ to any tangent vector at the corresponding point $t \in M$. The complete parameter space $M$ is called a Kodaira moduli space.

About 14 years later Penrose [17] studied the Kodaira moduli space $M$ of rational curves $X_{t} \simeq \mathbb{C P}^{1}$ embedded into a 3-dimensional complex manifold $Y$ with normal bundle $N_{t} \cong \mathbb{C}^{2} \otimes \mathfrak{O}(1)$. His striking conclusion was that 4 -fold $M$ comes equipped canonically with an induced half-flat conformal structure. This Penrose idea to study geometric structures canonically induced on Kodaira moduli spaces led to a radically new way of solving of a number non-linear differential equations in the form of constraints on curvature and torsion tensors of an affine connection thereby revealing deep interconnections between multidimensional complex analysis and differential geometry (see, e.g., the books $[1,3,4,7,11,18,21]$ and references cited therein). Instead of analysing some particular Kodaira moduli spaces (as is normally done in twistor theory, where moduli spaces of rational curves and quadrics with specific normal bundles have been only considered), we attempt in this paper to find sufficient conditions for a general Kodaira moduli space to have a canonically induced family of torsion-free affine connections satisfying some natural integrabily conditions. We do it through the analysis of the second floors of towers of infinitesimal neighbourhoods generated by embeddings $t \hookrightarrow M$ and

Received by the editors October 19, 1994.

1991 Mathematics Subject Classification. Primary 32G10, 32L25, 53B05, 53B10. 
$X_{t} \hookrightarrow Y$. Probably the most interesting aspect of the emerging theory is that both the Kodaira condition for existence of an induced complex manifold structure and the conditions for existence of an induced geometric structure on $M$ have the form of vanishing of some cohomology groups associated with normal bundles. As an application we show how several "curved twistor" constructions follow from one and the same general theorem, thus merging several fragments into one picture.

\section{KodAira's EXISTENCE THEOREM}

Let $Y$ and $M$ be complex manifolds and let $\pi_{1}: Y \times M \longrightarrow Y$ and $\pi_{2}: Y \times M \longrightarrow M$ be the natural projections. An analytic family of compact submanifolds of the complex manifold $Y$ with the moduli space $M$ is a complex submanifold $F \hookrightarrow$ $Y \times M$ such that the restriction of the projection $\pi_{2}$ on $F$ is a proper regular map (regularity means that the rank of the differential of $\left.\nu \equiv \pi_{2}\right|_{F}: F \longrightarrow M$ is equal at every point to $\operatorname{dim} M$ ). Thus the family $F$ has the structure of a double fibration

$$
Y \stackrel{\mu}{\longleftarrow} F \stackrel{\nu}{\longrightarrow} M
$$

where $\left.\mu \equiv \pi_{1}\right|_{F}$. For each $t \in M$ the compact complex submanifold $X_{t}=\mu \circ$ $\nu^{-1}(t) \hookrightarrow Y$ is said to belong to the family $M$. If $F \hookrightarrow Y \times M$ is an analytic family of compact submanifolds, then, for any $t \in M$, there is a natural linear map

$$
\mathrm{k}_{t}: T_{t} M \longrightarrow H^{0}\left(X_{t}, N_{t}\right),
$$

from the tangent space at $t$ to the vector space of global holomorphic sections of the normal bundle $N_{t}=\left.T Y\right|_{X_{t}} / T X_{t}$ to the submanifold $X_{t} \hookrightarrow Y$.

An analytic family $\left\{X_{t} \hookrightarrow Y \mid t \in M\right\}$ of compact submanifolds is called complete if the Kodaira map $\mathrm{k}_{t}$ is an isomorphism at each point $t$ in the moduli space $M$. It is called maximal at a point $t_{0} \in M$, if, for any other analytic family $\left\{X_{\tilde{t}} \hookrightarrow Y \mid \tilde{t} \in \tilde{M}\right\}$ of compact complex submanifolds such that $X_{t_{0}}=X_{\tilde{t}_{0}}$ for a point $\tilde{t}_{0} \in \tilde{M}$, there exists a neighbourhood $\tilde{U} \subset \tilde{M}$ of $\tilde{t}_{0}$ and a holomorphic map $f: \tilde{U} \longrightarrow M$ such that $f\left(\tilde{t}_{0}\right)=t_{0}$ and $X_{f\left(\tilde{t}^{\prime}\right)}=X_{\tilde{t}^{\prime}}$ for each $\tilde{t}^{\prime} \in \tilde{U}$. The family $\left\{X_{t} \hookrightarrow Y \mid t \in M\right\}$ is called maximal if it is maximal at each point $t$ in the moduli space $M$.

In 1962 Kodaira proved that if $X \hookrightarrow Y$ is a compact complex submanifold with normal bundle $N$ such that $H^{1}(X, N)=0$, then $X$ belongs to the complete analytic family $\left\{X_{t}: t \in M\right\}$ of compact submanifolds $X_{t}$ of $Y$. The family is maximal and its moduli space is of complex dimension $\operatorname{dim}_{\mathbb{C}} H^{0}(X, N)$.

\section{Alpha subspaces of moduli spaces}

Let $\left\{X_{t} \hookrightarrow Y \mid t \in M\right\}$ be a complete family of compact complex submanifolds. For any point $y \in Y^{\prime} \equiv \bigcup_{t \in M} X_{t}$, there is an associated subset $\nu \circ \mu^{-1}(y)$ in $M$. It is easy to show that such a subset is always an analytic subspace of $M$. Moreover, if the natural evaluation map

$$
H^{0}\left(X_{t}, N_{t}\right) \otimes \mathfrak{O}_{X_{t}} \longrightarrow N_{t}
$$

is an epimorphism for all $t \in \nu \circ \mu^{-1}(y)$, then the subspace $\nu \circ \mu^{-1}(y) \subset M$ has no singularities, i.e. it is a submanifold. In general, we denote the manifold content of $\nu \circ \mu^{-1}(y)$ by $\alpha_{y}$ and, following the terminology of the seminal paper by Penrose [17], call it an alpha subspace of $M$. 


\section{Two NATURAL ShEAVES ON MODUli SPACE}

Let $F \hookrightarrow Y \times M$ be a complete analytic family of compact submanifolds. The Kodaira theorem asserts the existence of a sheaf isomorphism $\mathrm{k}: T M \longrightarrow \nu_{*}^{0}\left(N_{F}\right)$, where $N_{F}$ denotes the normal bundle of $F$ in $Y \times M$. It is easy to see that $\mathrm{k}^{-1}$ induces canonically the following morphisms of $\mathfrak{O}_{M}$-modules

$$
\begin{array}{cccc}
\phi_{1}: & \nu_{*}^{0}\left(N_{F} \otimes S^{2}\left(N_{F}^{*}\right)\right) & \longrightarrow & T M \otimes S^{2}\left(\Omega^{1} M\right), \\
\phi_{2}: & \nu_{*}^{0}\left(N_{F} \otimes N_{F}^{*}\right) & \longrightarrow & T M \otimes \Omega^{1} M,
\end{array}
$$

whose images we denote by $\Lambda$ and $\Theta$ respectively. Thus, the locally free sheaves $T M \otimes S^{2}\left(\Omega^{1} M\right)$ and $T M \otimes \Omega^{1} M$ on any Kodaira moduli space $M$ come equipped canonically with $\mathfrak{O}_{M}$-submodules $\Lambda$ and, respectively, $\Theta$ which may fail to be locally free in general.

\section{VeCtor BundLES ON $Y$ AND AFFine CONNECTIONS ON $M$}

Let $\left\{X_{t} \hookrightarrow Y \mid t \in M\right\}$ be a complete family of compact complex submanifolds. After the work by Ward [19] on instanton solutions of Yang-Mills equations much attention has been paid to holomorphic vector bundles $E$ on $Y$ which are trivial on submanifolds $X_{t}$ for all $t \in M$. Their geometric role is that they generate vector bundles on $M$ together with linear connections which are integrable on alpha subspaces of $M$ (see, e.g., $[11,21]$ ). In this subsection we find a geometric meaning of holomorphic vector bundles $E$ on $Y$ which, when restricted to $X_{t}, t \in M$, are canonically isomorphic to the normal bundle $N_{t}$ of the embedding $X_{t} \hookrightarrow Y$, i.e. $\left.E\right|_{X_{t}}=N_{t}$.

Theorem 1. Let $\left\{X_{t} \hookrightarrow Y \mid t \in M\right\}$ be a complete family of compact submanifolds. Suppose there is a holomorphic vector bundle $E$ on $Y$ such that $\left.E\right|_{X_{t}}=N_{t}$ for all $t$ in some domain $M_{0} \subseteq M$. If $H^{0}\left(X_{t}, N_{t} \otimes S^{2}\left(N_{t}^{*}\right)\right)=H^{1}\left(X_{t}, N_{t} \otimes S^{2}\left(N_{t}^{*}\right)\right)=0$ for each $t \in M_{0}$, then $M_{0}$ comes equipped canonically with an induced torsion-free affine connection such that, for every $y \in Y^{\prime}$, the associated alpha subspace $\alpha_{y} \cap M_{0}$ is totally geodesic.

Let $\left(X, \mathfrak{O}_{X}\right)$ be an analytic subspace of a complex manifold $\left(Y, \mathfrak{O}_{Y}\right)$ defined by the sheaf of ideals $J \subset \mathfrak{O}_{Y}$. The $m$ th-order infinitesimal neighborhood of $X$ in $Y$ is the ringed space $X^{(m)}=\left(X, \mathfrak{O}_{X}^{(m)}\right)$ with the structure sheaf $\mathfrak{O}_{X}^{(m)}=\mathfrak{O}_{Y} / J^{m+1}$. With the $(m+1)$ th-order infinitesimal neighborhood there is naturally associated an mth-order conormal sheaf of $\mathfrak{O}_{X}^{(m)}$-modules $\mathfrak{O}_{X}^{(m)}\left(N^{*}\right)=J / J^{m+2}$ (cf. [6]). By construction, $\mathfrak{O}_{X}^{(0)}\left(N^{*}\right)$ is the usual conormal sheaf $N^{*}$, while $\mathfrak{O}_{X}^{(1)}\left(N^{*}\right)$ fits into the exact sequence of $\mathfrak{O}_{X}^{(1)}$-modules

$$
0 \longrightarrow S^{2}\left(N^{*}\right) \longrightarrow \mathfrak{O}_{X}^{(1)}\left(N^{*}\right) \longrightarrow N^{*} \longrightarrow 0 .
$$

When $X$ is a point in $Y$, then $\left(\mathfrak{O}_{X}^{(0)}\left(N^{*}\right)\right)^{*}$ is identical to the tangent space $T_{X} Y$ at $X \in Y$, while $\left(\mathfrak{D}_{X}^{(1)}\left(N^{*}\right)\right)^{*}$ is usually denoted by $T_{X}^{[2]} Y$ and called second-order tangent space.

Proof of Theorem 1. By assumption, there is a holomorphic vector bundle on $Y$ such that $\left.E\right|_{X_{t}}=N_{t}$ for any given $t \in M$. The restriction of its dual to the first 
order infinitesimal neighbourhood of $X_{t}$ in $Y$ is an $\mathfrak{O}_{X_{t}}^{(1)}$-module which fits into the exact sequence [11]

$$
\left.\left.\left.0 \longrightarrow N_{t}^{*} \otimes E^{*}\right|_{X_{t}} \stackrel{i}{\longrightarrow} E^{*}\right|_{X_{t}^{(1)}} \longrightarrow E^{*}\right|_{X_{t}} \longrightarrow 0 .
$$

Therefore, one obtains an extension

$$
\left.0 \longrightarrow S^{2}\left(N_{t}^{*}\right) \longrightarrow E^{*}\right|_{X_{t}^{(1)}} / i\left(\wedge^{2} N^{*}\right) \longrightarrow N_{t}^{*} \longrightarrow 0 .
$$

It is straightforward to show using local coordinate representations of all objects involved [13] that the difference

$$
\left[\mathfrak{O}_{X_{t}}^{(1)}\left(N^{*}\right)-\left.E^{*}\right|_{X_{t}^{(1)}} / i\left(\wedge^{2} N^{*}\right)\right] \in \operatorname{Ext}_{\mathfrak{O}_{X}^{(1)}}\left(N^{*}, S^{2}\left(N^{*}\right)\right)
$$

is a locally free $\mathfrak{O}_{X_{t}}$-module which compares to 2nd order two embeddings, $X_{t} \hookrightarrow$ $Y$ and $\left.X_{t} \hookrightarrow E\right|_{X_{t}}$, which are equivalent at 0 th and 1st orders. We denote its dual sheaf by $\Delta_{X_{t}}^{[2]}(Y, E)$. The crucial property of this creature is that there is a commutative diagram,

(1)

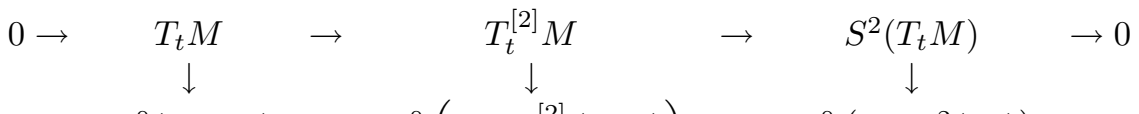

$$
\begin{aligned}
& 0 \rightarrow H^{0}\left(X_{t}, N_{t}\right) \rightarrow H^{0}\left(X_{t}, \Delta_{X_{t}}^{[2]}(Y, E)\right) \rightarrow H^{0}\left(X_{t}, S^{2}\left(N_{t}\right)\right)
\end{aligned}
$$

which relates the first floors of two towers of infinitesimal neighbourhoods generated by embeddings $t \hookrightarrow M_{0}$ and $X_{t} \hookrightarrow Y$. The verification is straightforward when one uses local coordinates [13]. Since, by assumption, $H^{1}\left(X_{t}, N_{t} \otimes S^{2}\left(N^{*}\right)\right)=0$, the exact sequence

$$
0 \longrightarrow N_{t} \longrightarrow \Delta_{X_{t}}^{[2]}(Y, E) \longrightarrow S^{2}\left(N_{t}\right) \longrightarrow 0
$$

splits. Moreover, its splitting is unique for the group $H^{0}\left(X_{t}, N_{t} \otimes S^{2}\left(N_{t}^{*}\right)\right)$ is also assumed to vanish. The commutative diagram (1) implies then a canonical splitting of the exact sequence for $T_{t}^{[2]} M$ which is the same thing as a torsion-free affine connection at $t$. This shows that the moduli space $M_{0}$ comes equipped canonically with an induced torsion-free affine connection. The fact that this connection is integrable on alpha surfaces is an easy exercise.

For any given relative deformation problem $X \hookrightarrow Y$, it is not difficult to identify a large class of holomorphic vector bundles $E$ on the ambient manifold $Y$ which have the property required by Theorem 1 . Indeed, take any holomorphic distribution $E \subset T Y$ on $Y$ which is transverse to $X$ and has rank equal to codim $X$. Then, for all $t$ in a sufficiently small neighbourhood $M_{0}$ of the point $t_{0} \in M$ corresponding to $X$, the submanifolds $X_{t} \hookrightarrow Y$ remain transverse to $E$, and one has a canonical isomorphism $\left.E\right|_{X_{t}}=N_{X_{t}}$.

Let us illustrate Theorem 1 by examples. The first one is just the original Penrose non-linear graviton construction [17]. Recall that Penrose established a one-to-one correspondence between "small" 4-dimensional complex Riemannian manifolds $M_{0}$ with self-dual Riemann tensor and 3-dimensional complex manifolds $Y$ equipped with the following data: (i) a submanifold $X \simeq \mathbb{C} P^{1}$ with normal bundle $N \simeq$ $\mathbb{C}^{2} \otimes \mathfrak{O}_{\mathbb{C} P^{1}}(1)$; (ii) a fibration $\pi: Y \longrightarrow X$; (iii) a global non-vanishing section of the "twisted" determinant bundle $\operatorname{det}\left(V_{\pi}\right) \otimes \pi^{*}(\mathfrak{O}(2))$, where $V_{\pi}$ is the vector bundle of $\pi$-vertical vector fields. Let us look at this data from the point of view 
of Theorem 1. The distribution $V_{\pi}$ is clearly transversal to $X$, and one easily checks that $H^{0}\left(X_{t}, N_{t} \otimes S^{2}\left(N_{t}^{*}\right)\right)=H^{1}\left(X_{t}, N_{t} \otimes S^{2}\left(N_{t}^{*}\right)\right)=0$. Therefore, by Theorem 1, the data (i) and (ii) imply that there is a torsion-free affine connection induced on $M_{0}$ which satisfies natural integrability conditions. What is the role of datum (iii)? It will be shown in section 6 that datum (iii) ensures that this induced connection is precisely the Levi-Civita connection of a holomorphic metric with self-dual Riemann tensor. Therefore, the non-linear graviton construction is one of the manifestations of the phenomenon envisaged by Theorem 1. Another manifestation is provided by relative deformation problems studied in the context of quaternionic geometry. Let $Y$ be a complex $(2 n+1)$-dimensional manifold equipped with a holomorphic contact structure which is a maximally non-degenerate rank $2 n$ holomorphic distribution $D \subset T Y$. Let $X$ be a rational curve embedded into $Y$ transversely to $D$ and with normal bundle $N=\mathbb{C}^{2 n} \otimes \mathfrak{O}(1)$. Then Theorem 1 says that the $4 n$-dimensional Kodaira moduli space $M_{0}$ comes equipped with a unique torsion-free affine connection satisfying natural integrability conditions. This is in accordance with well-known results in twistor theory. The case $n=1$ has been studied by Ward [20] and Hitchin [8] who showed that $M_{0}$ has an induced complex Riemannian metric satisfying self-dual Einstein equations with non-zero scalar curvature. The case $n \geq 2$ has been investigated by Bailey and Eastwood [2], LeBrun [10], and Pedersen and Poon [15] who proved that the moduli space $M_{0}$ comes equipped canonically with a torsion-free connection compatible with the induced (complexified) quaternionic structure on $M$.

\section{Families of affine Connections on moduli spaCes}

What happens when $H^{0}\left(X_{t}, N_{t} \otimes S^{2}\left(N_{t}^{*}\right)\right) \neq 0$ ? In this case, as we noted in section 3 , the locally free $\mathfrak{O}_{M}$-module $T M \otimes S^{2}\left(\Omega^{1} M\right)$ comes equipped with a nonzero $\mathfrak{O}_{M}$-submodule $\Lambda$. If $\nabla_{1}: T M \longrightarrow T M \otimes \Omega^{1} M$ and $\nabla_{2}: T M \longrightarrow T M \otimes \Omega^{1} M$ are two torsion-free affine connections on $M$, then their difference, $\nabla_{1}-\nabla_{2}$, is a global section of $T M \otimes S^{2}\left(\Omega^{1} M\right)$. We say that $\nabla_{1}$ and $\nabla_{2}$ are $\Lambda$-equivalent, if

$$
\nabla_{1}-\nabla_{2} \in H^{0}(M, \Lambda)
$$

We define a $\Lambda$-connection on $M$ as a collection of ordinary torsion-free affine connections $\left\{\nabla_{i} \mid i \in I\right\}$ on an open covering $\left\{U_{i} \mid i \in I\right\}$ of $M$ which, on overlaps $U_{i j}=U_{i} \cap U_{j}$, have their differences in $H^{0}\left(U_{i j}, \Lambda\right)$. Locally, a $\Lambda$-connection is the same thing as a $\Lambda$-equivalence class of affine connections, but globally they are different - the obstruction for existence of a $\Lambda$-connection on $M$ lies in $H^{1}\left(M, T M \otimes S^{2}\left(\Omega^{1} M\right) / \Lambda\right)$, while the obstruction for existence of a $\Lambda$-equivalence class of affine connections is an element of $H^{1}\left(M, T M \otimes S^{2}\left(\Omega^{1} M\right)\right)$. A submanifold of $M$ is said to be totally geodesic relative to a $\Lambda$-connection if it is totally geodesic relative to each of its local representatives $\nabla_{i}$.

Theorem 2. Let $\left\{X_{t} \hookrightarrow Y \mid t \in M\right\}$ be a complete family of compact submanifolds. Suppose there is a holomorphic vector bundle $E$ on $Y$ such that $\left.E\right|_{X_{t}}=N_{t}$ for all $t$ in some domain $M_{0} \subseteq M$. If $H^{1}\left(X_{t}, N_{t} \otimes S^{2}\left(N_{t}^{*}\right)\right)=0$ for each $t \in M_{0}$, then $M_{0}$ comes equipped canonically with an induced $\Lambda$-connection such that, for every $y \in Y^{\prime}$, the associated alpha subspace $\alpha_{y} \cap M_{0}$ is totally geodesic.

Proof. Since $H^{1}\left(X_{t}, N_{t} \otimes S^{2}\left(N_{t}^{*}\right)\right)=0$, the exact sequence (2) splits, and any such splitting, i.e. an $\mathfrak{O}_{X_{t}}$-morphism $\Delta_{X_{t}}^{[2]}(Y, E) \rightarrow N_{t} \oplus S^{2}\left(N_{t}^{*}\right)$, induces an associated 
(via commutative diagram (1)) splitting of the exact sequence for the second-order tangent space $T_{t}^{[2]} M$ which in turn induces a torsion-free affine coonection at $t \in$ $M_{0}$. The set of all splittings of (2) is a principal homogeneous space for the group $H^{0}\left(X_{t}, N_{t} \otimes S^{2}\left(N_{t}^{*}\right)\right)$. Therefore, the set of induced torsion-free affine connections at $t$ is a principal homogeneous space for the group $\Lambda_{t}$.

Next we study another equivalence class of affine connections which is often induced on Kodaira moduli spaces. Let sym denote the natural projection

$$
\text { sym : } T M \otimes \Omega^{1} M \otimes \Omega^{1} M \rightarrow T M \otimes S^{2}\left(\Omega^{1} M\right) .
$$

Associated with a subsheaf $\Xi \subset T M \otimes \Omega^{1} M$ there is a concept of $\Xi$-connection on $M$ which is, by definition, a collection of ordinary torsion-free affine connections $\left\{\nabla_{i} \mid i \in I\right\}$ on an open covering $\left\{U_{i} \mid i \in I\right\}$ of $M$ which, on overlaps $U_{i j}=U_{i} \cap U_{j}$, have their differences in $H^{0}\left(U_{i j}, \operatorname{sym}\left(\Xi \otimes \Omega^{1} M\right)\right)$. If $\Xi$ happens to be the structure sheaf $\mathfrak{O}_{M}$ embedded diagonally into $T M \otimes \Omega^{1} M$, then a $\Xi$-connection is nothing but a torsion-free projective connection on $M$.

Theorem 3. Let $\left\{X_{t} \hookrightarrow Y \mid t \in M\right\}$ be a complete family of compact submanifolds. Suppose there is a holomorphic vector bundle $E$ on $Y$ such that $\left.E\right|_{X_{t}}=N_{t}$ for all $t$ in some domain $M_{0} \subseteq M$. If $H^{1}\left(X_{t}, N_{t} \otimes N_{t}^{*}\right)=0$ for each $t \in M_{0}$, then $M_{0}$ comes equipped canonically with an induced $\Xi$-connection such that, for every $y \in Y^{\prime}$, the associated alpha subspace $\alpha_{y} \cap M_{0}$ is totally geodesic.

This theorem is proved by a slight modification of the construction used in the proof of Theorem 1 [13]. Note that the condition $H^{1}\left(X_{t}, N_{t} \otimes N_{t}^{*}\right)=0$ only says that $N_{t}$ is a rigid vector bundle on $X_{t}$. There is an important case when the requirement $\left.E\right|_{X_{t}}=N_{t}$ can be safely replaced by a weaker condition that vector bundles $\left.E\right|_{X_{t}}$ and $N_{t}$ are simply equivalent to each other, $\left.E\right|_{X_{t}} \simeq N_{t}$, which means that they define the same cohomology class in $H^{1}\left(X_{t}, G L\left(p, \mathfrak{O}_{X_{t}}\right)\right), p=\operatorname{codim} X$.

Theorem 4. Let $\left\{X_{t} \hookrightarrow Y \mid t \in M\right\}$ be a complete family of compact 1-codimensional submanifolds. Suppose there is a holomorphic line bundle $E$ on $Y$ such that $\left.E\right|_{X_{t}} \simeq N_{t}$ for all $t$ in some domain $M_{0} \subseteq M$. If $H^{1}\left(X_{t}, \mathfrak{O}_{X_{t}}\right)=0$ for each $t \in M_{0}$, then $M_{0}$ comes equipped canonically with an induced torsion-free projective connection such that, for every $y \in Y^{\prime}$, the associated alpha subspace $\alpha_{y} \cap M_{0}$ is totally geodesic.

Proof. Fix any isomorphism $\phi:\left.E\right|_{X_{t}} \longrightarrow N_{t}$ and apply Theorem 4 to construct a projective connection $\nabla$ on $M_{0}$. Due to compactness of $X_{t}$, the isomorphism $\phi$ is defined up to multiplication by a non-zero constant. The point is that the construction of $\nabla$ sketched in the proof of Theorem 1 is invariant under such a transformation which shows that $\nabla$ is actually independent on a particular choice of the isomorphism $\phi$ used in its construction.

Corollary 5. Let $\left\{X_{t} \hookrightarrow Y \mid t \in M\right\}$ be a complete family of compact 1-codimensional submanifolds. If $H^{1}\left(X_{t_{0}}, \mathfrak{O}_{X_{t_{0}}}\right)=0$ for some $t_{0} \in M$, then there is an open neigbourhood $M_{0}$ of $t_{0}$ which comes equipped canonically with an induced torsionfree projective connection such that, for every $y \in Y^{\prime}$, the associated alpha subspace $\alpha_{y} \cap M_{0}$ is totally geodesic.

Proof. By semi-continuity principle, there is an open neighbourhood $U$ of $t_{0}$ such that $H^{1}\left(X_{t}, \mathfrak{O}_{X_{t}}\right)=0$ for each $t \in U$. If $L$ is the divisor line bunlde of $X_{t_{0}}$, then 
$\left.L^{*}\right|_{X_{t}} \simeq N_{t}$ for all $t$ in probably smaller neighbourhood $M_{0}$. This fact combined with Theorem 4 implies the desired result.

An independent (and more effective from the viewpoint of computing induced projective structures) proof of this Corollary is given in [14].

\section{Fibred COMPLEX MANifolds}

Suppose that the ambient manifold $Y$ has the structure of a holomorphic fibration over its compact submanifold $X$, i.e. there is a submersive holomorphic map $\pi: Y \longrightarrow X$. If $H^{1}(X, N)=0$, then, by Kodaira's theorem, $X$ belongs to the complete family $\left\{X_{t} \hookrightarrow Y \mid t \in M\right\}$ of compact submanifolds. The submanifold $X$ is transverse to the distribution $V_{\pi}$ of $\pi$-vertical vector fields on $Y$, and so is $X_{t}$ for every $t$ in some neighbourhood $M_{0}$ of the point $t_{0} \in M$ which corresponds to $X$. Thus $Y$ has the structure of a holomorphic fibration $Y \longrightarrow X_{t}$ for $t \in M_{0}$. If also $H^{1}\left(X_{t}, N_{t} \otimes S^{2}\left(N_{t}^{*}\right)\right)=0$, then, by Theorem 2, the moduli space comes equipped with a family of induced torsion-free affine connections. Holonomy groups of these induced connections can be estimated with surprising ease.

Theorem 6. Let $\nabla$ be an induced connection on the moduli space $M_{0}$. If the function

$$
f: t \longrightarrow \operatorname{dim} H^{0}\left(X_{t}, N_{t} \otimes N_{t}^{*}\right)
$$

is constant on $M_{0}$, then the holonomy algebra of $\nabla$ is a subalgebra of the finitedimensional Lie algebra $H^{0}\left(X, N \otimes N^{*}\right)$. If in addition there is a holomorphic line bundle $L$ on $X$ such that the bundle $\pi^{*}(L) \otimes \operatorname{det} V_{\pi}^{*}$ admits a nowhere vanishing holomorphic section, then the holonomy algebra of $\nabla$ is a subalgebra of $H^{0}\left(X, N \otimes_{0} N^{*}\right)$, where $\otimes_{0}$ denotes trace-free tensor product.

This theorem follows from the correspondence between third floors of the two towers of infinitesimal neighbourhoods of embeddings $t \hookrightarrow M$ and $X_{t} \hookrightarrow Y$ which has been studied in [12].

Examples. 1. Let $X=\mathbb{C P}^{1}$ be the projective line embedded into a 3-dimensional complex manifold $Y$ with normal bundle $N \simeq \mathbb{C}^{2} \otimes \mathfrak{O}(1)$. If $Y$ has the structure of a holomorphic fibration over $X$, then Theorem 1 says that there is an induced connection $\nabla$ on the moduli space $M_{0}$. By Theorem 6 , the holonomy algebra of $\nabla$ is contained in $H^{0}\left(X, N \otimes N^{*}\right)=M_{2,2}(\mathbb{C}) \subset c o(4, \mathbb{C})$, where $M_{2,2}(\mathbb{C})$ is the space of $2 \times 2$ complex matrices and $\operatorname{co}(4, \mathbb{C})=\operatorname{sl}(2, \mathbb{C})+\operatorname{sl}(2, \mathbb{C})+\mathbb{C}$ the complexified conformal algebra. Since $\nabla$ is torsion-free, this fact implies that $\nabla$ is a complex Weyl connection on the 4-dimensional complex conformal manifold $M_{0}$ which has the anti-self-dual parts of the Weyl tensor and the antisymmetrized Ricci tensor vanishing and satisfies the Einstein-Weyl equations. In fact any such connection arises locally in this way $[17,5,16]$.

2. Let the pair $\left(X=\mathbb{C P}^{1}, Y\right)$ be the same as in Example 1 and assume that the bundle $\operatorname{det} V_{\pi}^{*} \otimes \pi^{*}(\mathfrak{O}(2))$ admits a nowhere vanishing holomorphic section. By Theorem 6 , the holonomy group of the induced connection $\nabla$ on the 4-dimensional parameter space $M_{0}$ is a subgroup of $S L(2, \mathbb{C}) \subset S O(4, \mathbb{C})=S L(2, \mathbb{C}) \times_{\mathbb{Z}_{2}} S L(2, \mathbb{C})$. This means that $\nabla$ is the Levi-Civita connection of a complex Riemannian metric on $M_{0}$ which is Ricci-flat and has the anti-self-dual part of the Weyl tensor vanishing. Again any such connection arises locally in this way [17].

3. Let $X=\mathbb{C P}^{1}$ be the projective line embedded into a $(2 k+1)$-dimensional complex manifold $Y$ with normal bundle $N \simeq \mathbb{C}^{2 k} \otimes \mathfrak{O}(1), k \geq 2$. The Kodaira moduli 
space $M$ is then a $4 k$-dimensional complex manifold possessing a complexified almost quaternionic structure. If $Y$ has the structure of a holomorphic fibration over $X$, then, by Theorems 1 and 6 , there is an induced connection $\nabla$ on $M_{0} \subset M$ with holonomy in $G L(2 k, \mathbb{C})$ which implies that $\nabla$ is a complexified Obata connection. It is not difficult to show using results of Bailey and Eastwood [2] that any local complexified Obata connection can be constructed in this way. A twistor characterization of real analytic Obata connections can be obtained from this holomorphic picture in the usual way (cf. [11]).

\section{REFERENCES}

1. T. N. Bailey and R. J. Baston (eds.), Twistors in mathematics and physics, Cambridge University Press, Cambridge, 1990. MR 91k:32033

2. T. N. Bailey and M. G. Eastwood, Complex paraconformal manifolds - their differential geometry and twistor theory, Forum Math., 3 (1991), 61-103. MR 92a:32038

3. R. J. Baston and M. G. Eastwood, The Penrose transform, Oxford University Press, Oxford, 1989. MR 92j:32112

4. A. Besse, Einstein manifolds, Springer, Berlin, 1987. MR 88f:53087

5. C. P. Boyer, A note on hyper-Hermitian four-manifolds, Proc. Amer. Math. Soc. 102 (1988), 157-164. MR 89c:53049

6. M. G. Eastwood and C. R. LeBrun, Fattening complex manifolds: curvature and KodairaSpencer maps, J. Geom. Phys. ,8 (1992), 123-146. MR 93d:32048

7. S. A. Huggett (ed.), Twistor theory, Marcel Dekker, 1994, to appear. MR 95f:00013

8. N. Hitchin, Complex manifolds and Einstein's equations, In: H. D. Doebner, et al. (eds.) Twistor geometry and non-linear systems, Springer, Berlin, Lect. Notes Math., vol. 970 (1982), 73-99. MR 84i:32041

9. K. Kodaira, A theorem of completeness of characteristic systems for analytic families of compact submanifolds of complex manifolds, Ann. Math., 75 (1962), 146-162. MR 24:A3665

10. C. LeBrun, Quaternionic-Kähler manifolds and conformal geometry, Math. Ann., 284 (1989), 353-376. MR 90e:53062

11. Yu. I. Manin, Gauge Field theory and Complex Geometry, Nauka, Moscow 1984.

12. S. A. Merkulov, Moduli of compact submanifolds of complex fibred manifolds, to appear in Math. Proc. Cambridge Phil. Soc.

13. _ Geometry of relative deformations I, In Twistor theory, Marcel Dekker, 1994, to appear. CMP 95:05

14. S. A. Merkulov and H. Pedersen, Geometry of relative deformations II, In Twistor theory, Marcel Dekker 1994, to appear. CMP 95:05

15. H. Pedersen and Y. S. Poon, Twistorial construction of quaternionic manifolds, In Proc. VIth Int. Coll. on Diff. Geom. , Cursos y Congresos, Univ. Santiago de Compostela, 61 (1989), 207-218. MR 91f:53040

16. H. Pedersen and A. Swann, Riemannian submersions, four-manifolds and Einstein-Weyl geometry, Proc. London Math. Soc. 66 (1993), 381-399. MR 94c:53061

17. R. Penrose, Non-linear gravitons and curved twistor theory, Gen. Rel. Grav. 7 (1976), 31-52. MR 55:11905

18. R. Penrose and W. Rindler, Spinors and space-time, Vol.2, Cambridge University Press, Cambridge, 1986. MR 88b:83003

19. R. S. Ward, On self-dual gauge fields, Phys. Lett. 61A (1977), 81-82. MR 56:2186

20. $ـ$ Self-dual space-times with cosmological constant, Commun. Math. Phys., 78 (1980), 1-17. MR 82g:83004

21. R. S. Ward and R. O. Wells, Jr., Twistor geometry and field theory, Cambridge University Press, Cambridge, 1990. MR 91b:32034

School of Mathematics and Statistics, University of Plymouth, Plymouth, Devon PL4 8AA, United Kingdom

Current address: Department of Pure Mathematics, Glasgow University, 15 University Gardens, Glasgow G12 8QW, United Kingdom

E-mail address: sm@maths.gla.ac.uk 